\title{
THE FORMATION AND EVOLUTION
}

\section{OF BLACK-HOLE BINARIES}

\author{
ROGER W. ROMANI \\ Department of Physics \\ Stanford University \\ Stanford, CA 94305-4060, U.S.A.
}

\section{Introduction}

The presence of accreting black holes ( $\mathrm{BH})$ among the $\mathrm{X}$-ray binaries has been recognized for many years. Traditionally, Cyg X-1 and the handful of other candidates have been thought of as cousins of the HMXB neutron star systems. Recent studies of the soft X-ray transients such as A 0620-00 have, however, shown that the dynamical evidence makes these low-mass systems very strong black-hole candidates. Further, analysis of the eventual end-states of various high-mass $\mathrm{X}$-ray binaries suggest that some could end as observable BH-pulsar binaries, although the first such system is yet to be discovered.

The study of black-hole binaries (BHB) is thus becoming a rich field observationally. Still, our theoretical understanding of the evolutionary tracks leading to such systems is somewhat rudimentary. Pioneering studies (e.g., Van den Heuvel \& Habets 1984; De Kool et al. 1987) have outlined some basic paths that could generate $\mathrm{BHB}$, in fairly strict analogy with the neutron star (NS) X-ray binary evolutions. However, a number of other scenarios for specific systems have been presented in the literature; we describe some of these in Section 2 below. A second important development has been the discovery of a large and diverse family of radio pulsar binaries offering a rich variety of evolutionary endpoints for models to match. This, coupled with observations of the pulsar population showing that the core collapse process is much more violent than previously believed, has substantially modified our picture of X-ray binary evolution. While similar observational constraints on black-hole formation processes are still far in the future, 
we can invoke some theoretical prejudice to contrast the genesis of NScontaining XRB with the equivalent black-hole systems (Section 3).

The differences in the evolution through core collapse and in the X-ray phase suggest that a number of peculiar types of black-hole binaries might have been formed from channels that are not available to the neutron star population. We describe a few of these briefly in Section 4. Finally, with the availability of simple analytic approximations for many of the important evolutionary processes, access to substantial computer time and a bit of hubris, it has become easy and popular to simulate the eventual fate of an entire population of primordial binaries. With such population sums one can estimate the probability of evolution into particular end states. Combined with estimates of lifetimes and visibility, these give 'predictions' that may be useful in guiding the interpretation of present surveys, as described in Section 5. Here we note that the broad conclusions of these models provide useful guidance, but that we will have to put the refining fire of observational tests to the scenarios before detailed prediction will merit much credence.

\section{Formation Models}

Several scenarios for the formation of black holes in binary systems have been proposed. These are similar to the evolutionary paths described for neutron star XRB by Webbink (1992). The basic difference is that here the primary has a mass greater than some critical value $M_{\mathrm{BH}}$, the mass beyond which the core cannot propagate a successful supernova explosion. The value of this mass is quite uncertain. The presence of NS in certain high-mass XRB argues that in some cases primaries with initial masses as high as $\sim 40 \mathrm{M}_{\odot}$ can eject their outer cores and envelopes in a successful supernova explosion, leaving $\sim 1.4 \mathrm{M}_{\odot}$ neutron stars (Van den Heuvel \& Habets 1984). Other authors, however, favor lower masses $M_{\mathrm{BH}} \sim 25 \mathrm{M}_{\odot}$ (Wheeler \& Shields 1976).

In fact it seems likely that $M_{\mathrm{BH}}$ may not be uniform in the Galaxy. In particular, at early times, at large galactocentric radii and in environments such as the Magellanic clouds, lower metalicity in regions of massive star formation result in lower opacities in the stellar envelopes. The result is a suppression of main-sequence winds and mass loss, which may favor the growth of a heavier core for a star of a given ZAMS mass. Conversely, in close interacting binaries, early transfer of envelope material and reduced escape velocity leading to enhanced stellar wind might increase mass loss and raise the required initial mass for black-hole formation. The picture adopted here is that mass loss is always increasingly important for stars approaching $M_{\mathrm{BH}} \sim 40 \mathrm{M}_{\odot}$. Lower-mass stars will also experience signif- 
icant mass loss well before core $\mathrm{H}$ exhaustion, and for the heavier stars even the outer layers of the core are shed before core collapse. A rough formula for the final pre-collapse core mass suggests that $\sim 7-15 \mathrm{M}_{\odot}$ typically remains of the massive progenitor (Romani 1994). We also picture the post-bounce shock wave stalling as it propagates through this massive core, leaving the outer layers of the core in dynamic collapse and trapping the neutrinos emitted from the hot central regions. Thus here the collapse is 'silent', resulting in no neutrino burst, little or no envelope ejection and little optical display. For very strong stellar winds or very close binaries it is possible that the heaviest stars' cores lose enough mass to avoid this silent collapse, restricting the $\mathrm{BH}$ formation range to intermediate masses (Woosley et al. 1993). Unless this effect dominates down to nearly $M_{\mathrm{BH}}$ this channel does not dominate the fate of the most massive stars and we ignore it in the discussion below. An attractive feature of our picture is that the resulting $\mathrm{BH}$ are in the $\sim 7-15 \mathrm{M}_{\odot}$ range, similar to the values observed for the present strong candidates.

We next summarize the scenarios that lead to binary black-hole systems. We shall see that even for steep IMFs ensuring that BH forming progenitors are rarer than the primaries that can form NS by a factor of $\sim 30$ (cf., Van den Heuvel 1993), the severe disruption associated with the supernova explosion greatly favors the survival of the BH systems. It is this effect which leads to a significant galactic population of certain types of $\mathrm{BH}$ binaries.

\section{1. "HE STAR CORE COLLAPSE"}

We distinguish three varieties of the model in which the black hole is formed from the He core remnant of a star with $M>M_{\mathrm{BH}}$ in a close binary with a companion that will eventually become the mass donor. In the 'direct' scenario which forms HMXB, two high-mass stars in a close binary evolve via strong stellar winds. After the primary reaches core collapse, a continued strong mass loss from the secondary can result in a bright wind fed system like Cyg X-1. This requires a secondary mass greater than $\sim 15-20 \mathrm{M}_{\odot}$. With a silent core collapse leading to a $\sim 7-15 \mathrm{M}_{\odot} \mathrm{BH}$, it seems unlikely that there will be many 'Be' systems accreting from the equatorial disk of a rapidly rotating secondary in an eccentric orbit. Instead, with a typical hole mass of $\sim 7-10 \mathrm{M}_{\odot}$, secondaries with mass $\sim 8-15 \mathrm{M}_{\odot}$ will have relatively weak stellar winds and will only initiate a bright $\mathrm{X}$-ray phase during a brief period of radius expansion resulting in 'incipient Roche lobe overflow' (Savonije 1983). This will likely be followed by a common envelope spiral in, which may leave either a close binary or a merged system. For the secondaries that do survive, core collapse leads to a neutron star (pulsar) + black 
hole system, which can remain bound after the supernova because of the relatively high mass of the black-hole companion. The pulsar will be a young object with a high field, spinning down and disappearing in $\sim 10^{7} \mathrm{y}$.

The second variety of He star core collapse can be labeled the Reverse Mass Transfer case (Narayan, Piran \& Shemi 1991). Here we start from a close ZAMS binary with $M_{1} \sim M_{2} \lesssim M_{\mathrm{BH}}$. The primary evolves, transferring mass to its companion via a strong stellar wind. When the primary undergoes a supernova explosion the system will remain bound in a modest fraction of the cases because of the large secondary mass, now $M_{2}>M_{\mathrm{BH}}$. As the secondary evolves, it transfers matter onto its companion in a classic HMXB with a blue supergiant donor. The mass transfer drives the initial neutron star to lower fields and shorter periods, keeping it above the pulsar death line. After the second core collapse one now has a binary with a $\mathrm{BH}$ in orbit around a mildly recycled pulsar. This pulsar will have a relatively long active phase - such systems are attractive targets for radio searches.

Finally, if the secondary is of low mass we have black-hole systems evolving in analogy to the He star model of LMXB formation (De Kool et al. 1987), for which $M_{2} \lesssim 1 \mathrm{M}_{\odot}$. Here, as the primary evolves through a giant phase, it comes into Roche lobe (or tidal) contact with the low-mass secondary, which becomes engulfed in a common envelope spiral-in. With adequate common envelope ejection efficiency (Taam, these proceedings) the system can emerge from this phase as a detached binary consisting of a massive $\mathrm{He}+\mathrm{Z}$ core and a low-mass star (Romani 1994). The silent core collapse poses no problem here and after evolution via angular momentum loss or radius expansion through core hydrogen exhaustion of the low mass companion, the resulting binary can have a long phase of mass transfer at a moderate rate. For secondary masses $\lesssim 8 \mathrm{M}_{\odot}$ this transfer will be stable.

\subsection{AIC OF A NEUTRON STAR}

The viability of NS formation via Accretion-Induced Collapse of a massive white dwarf (e.g., Nomoto \& Kondo 1991) is still the subject of some debate, but AIC seems an attractive model for the formation of certain LMXB and wide radio pulsar binary systems. There has been discussion of accretion driving a neutron star above its maximum stable mass (Chevalier 1989), which might be as low as $\sim 2 \mathrm{M}_{\odot}$ for some soft equations of state (Brown et al. 1992).

One arena where this might happen is in a two-stage collapse of a massive core (Chevalier 1989). After core collapse and the formation of a protoneutron star, a significant phase of late infall driven by the reverse shock in the progenitor envelope might push the neutron star above the maximum mass. There are some problems with this being the primary formation chan- 
nel for the binary BH systems that we do see. For one, the resulting holes would be light, typically $\sim 3 \mathrm{M}_{\odot}$, in contrast to the $\sim 10 \mathrm{M}_{\odot}$ estimated for the strong $\mathrm{BH}$ candidates. In addition the neutrino burst, velocity kick and mass ejection associated with the original supernova event make it very difficult for the system to survive as a detached binary, as seen below. So it seems unlikely that this mechanism can contribute many interacting $\mathrm{BH}$ binaries.

Alternatively, steady Roche lobe over-flow in an LMXB might be sufficient to effect the NS collapse. However, there does not seem to be much parameter space accessible to this channel if $\sim 1 \mathrm{M}_{\odot}$ of accreted mass is needed. If the secondary is any lighter than $\sim 1.5 \mathrm{M}_{\odot}$ it will not be able to donate the needed mass, while if it is heavier then $\sim 1.8 \mathrm{M}_{\odot}$ transfer will tend to runaway, leading to high $\dot{M}$ and little mass acceptance.

However, if the secondary is substantially more massive than the neutron star, the runaway to CE may push the effective $\dot{M}$ on the neutron star to very high values. AIC under this 'hypercritical accretion', where the accretion luminosity is carried off by neutrinos, may provide an interesting alternative channel (Chevalier 1993). More work on the flow around the embedded NS is however needed to see if the transition to hypercritical accretion actually takes place. Certainly in the case of the NS-NS binaries and the NS massive WD binaries such as PSR $0655+54$, only very modest mass accretion can have occurred (from the presence of recycled pulsars and from component mass estimates) so in many cases the transition to high value of $\dot{M}$ will not be made. Chevalier, in fact, shows that the transition to the hypercritical solution may most easily occur for $\mathrm{CE}$ in relatively dense envelopes. For these $\mathrm{CE}$ phases, initiating at small radius, it seems likely that complete spiral-in will result. This leads to the final AIC channel, described by Podsiadlowski (these proceedings): collapse in the core of a TŻO. Again viability is not clear, but the model has some attractive features, if the remnants of the disrupted TŻO envelope at the end of the accretion phase can form a low mass companion star. If such a star is formed and its nuclear evolution or the system angular momentum loss initiates transfer onto the $\mathrm{BH}$, a low-mass black-hole binary may be formed.

\subsection{TRIPLE SCENARIOS FOR LMBHB PRODUCTION}

A close relative of this last model is the scenario created for the low-mass black-hole binary A 0620-00 by Eggleton \& Verbunt (1986). In this 'Triple System' picture, a high-mass initial binary in a hierarchical triple with a distant low-mass companion evolves into a $\mathrm{HMXB}$ containing a $\mathrm{BH}$, while retaining the distant $\sim 1 \mathrm{M}_{\odot}$ star. After the MXRB secondary evolves, a $\mathrm{CE}$ forms, and the resulting spiral-in and final nuclear evolution causes 
the merged product with a compact core to expand to very large radii. The result is that the distant low-mass companion also enters the common envelope. However, with its large orbital energy, it may survive spiral-in to create a system like A $0620-00$, a BH in a close orbit with a low mass, main sequence companion. If one adds the possibility of AIC in the TŹO product of the initial spiral-in, then a classic NS-containing MXRB might suffice for the inner binary system.

\section{Contrast with NS Systems}

Even though the basic evolutionary paths outlined above have parallels in NS-XRB evolution, the evolution of BHB should differ from that of the NSsystems in a number of important ways. First, the higher-mass primaries will have especially high mass loss rates $\dot{M}$ prior to core collapse. Indeed, estimates of single-star mass loss (Woosley et al. 1993) suggest that wind loss can lead to bare He cores at collapse for $M>25 \mathrm{M}_{\odot}$, providing a source of $\mathrm{SN}$ Ibc, although these may also be largely formed via binary-induced wind loss (Podsiadlowski et al. 1992). In any case the high-mass BH-producing primaries in close binaries will lose substantial mass on the main sequence. This should assist the survival of low-mass companions in the CE phase, since the high gravity of the massive core and the relatively low envelope mass will require lower $\mathrm{CE}$ efficiencies and smaller initial orbits to survive the CE phase. Indeed, MS mass loss is essential in allowing CE formation of a close binary at high initial mass ratios. A second interesting possibility is that for sufficiently strong winds, the primary may never become a red giant, instead evolving directly to a WR-star phase and then making a silent collapse to a BH. Romani (1992) argued that this may occur in high-metalicity regions such as the galactic center and may give observable $\mathrm{X}$-ray binaries after the secondary evolves to contact (WR-evolution).

The second important difference lies in the core collapse itself. New estimates of initial pulsar velocities indicate that $\mathrm{SN}$ events give new-born neutron stars a typical kick velocity of $v_{\mathrm{rms}} \sim 450 \mathrm{~km} \mathrm{~s}^{-1}$, albeit with a wide distribution around this value (Lyne \& Lorimer 1994). At a minimum, neutron star forming cores must eject $\sim 1-5.5 \mathrm{M}_{\odot}$ to leave a $\sim 1.4 \mathrm{M}_{\odot}$ compact object. The attrition of binary systems with low-mass companions due to the Blaauw mechanism will be severe; in fact, Yamaoka et al. (1993) compute that kick velocities of order $v_{\text {rms }}$ above are need to simply keep some of the observed NS-NS systems bound. The BH systems, we have argued, have a silent collapse with no kick or mass ejection. They should therefore have a much better chance of retaining a low-mass companion after collapse. Even though the corresponding initial binaries with very high mass ratio may be formed rarely, after passing through the $\mathrm{CE}$ and core collapse phases, 
they will gain dramatically in the galactic population compared to their NS cousins. The test of this picture lies in the space velocities (and galactic scale heights) of the systems; NS-LMXB should have typical velocities $\sim 200 \mathrm{~km} \mathrm{~s}^{-1}$, while the BH X-ray transients should have $v<50 \mathrm{~km} \mathrm{~s}^{-1}$ and low $\langle z\rangle$. Differences should exist for the high-mass systems, as well. Even here, the large NS kicks cause disruption and/or merger for $\sim 2 / 3$ of the HMXBs; the rarer BH systems should thereby increase in representation.

We note that the presence of kicks does confer one advantage on the NS systems. When the secondary has a mass $8 \mathrm{M}_{\odot}<M_{2}<15 \mathrm{M}_{\odot}$, the SN mass loss and core kick tends to leave the resulting system in eccentric orbits; with kicks opposite the orbital velocity the semi-major axis will in fact shrink. NS with such close periastron approaches and these lower-mass companions will be able to spin up the secondary via tidal effects, generate an equatorial disk and thereby achieve a substantial phase of episodic accretion in a Be/X-ray binary. Black-hole collapse with a secondary in the same mass range will not only not generate an eccentric orbit, but will likely suffer runaway mass transfer when accretion starts since $M_{2} \gtrsim M_{\mathrm{BH}}$. This gives only a short X-ray bright phase as noted above.

The next important difference lies in the fate after the initial compact object is formed. When the secondary can itself form a neutron star, the attrition during core collapse is again severe for the neutron star systems: depending on the post-CE period distribution, roughly $1 / 30$ of these survive to become NS-NS binaries detectable by their recycled radio pulsars. Blackhole systems, having the $\mathrm{SN}$ occur with a $\sim 7-10 \mathrm{M}_{\odot}$ companion, will survive $\sim 1 / 3$ of the time, thereby enhancing the fraction of BH-NS binaries.

When the secondary has $2 \mathrm{M}_{\odot} \lesssim M_{2} \lesssim 10 \mathrm{M}_{\odot}$, transfer onto a neutron star primary will be unstable, leading to CE. In some cases, these systems may survive as detached binaries to form the PSR+massive WD systems (Bhattacharya, these proceedings). In any case, though, these will have a short bright $\mathrm{X}$-ray phase. However, when the accretor is a $\mathrm{BH}$ the mass ratio is favorable and stable accretion can proceed until the secondary finishes its evolution. X-ray sources with companion masses in the $3-5 \mathrm{M}_{\odot}$ range are likely to harbour BH, as in LMC X-3 (Cowley et al. 1983). Thus black-hole $\mathrm{X}$-ray binaries will not have the secondary mass gap of the NS XRB, but will instead have the 'gap' described above for $M_{2} \sim 8-15 \mathrm{M}_{\odot}$, and many fewer spun up Be-star companions.

Finally, for low-mass secondaries the LMXB statistics and luminosities indicate that some sort of accelerated evolution reduces the lifetime below the time scale for angular-momentum loss. Comparison with survival rates through $\mathrm{SN}$ in population computations suggests that $\tau_{\mathrm{ev}} \sim$ $310^{7} \mathrm{y}\left(\left\langle v_{\mathrm{rms}}\right\rangle / 100 \mathrm{~km} \mathrm{~s}^{-1}\right)$ to match the observed LMXB population, i.e., lifetimes of $\sim 10^{8} \mathrm{y}$. In contrast, for the BH LMXB quiescence accretion 
rates estimated from hot-spot fluxes seem more in line with rates predicted from $\dot{J}$ evolution. Thus these systems will have long $\gtrless^{9}$ y mass transfer lifetimes. However, the low secondary mass ratios $q \lesssim 0.1$, possibly abetted by the lack of soft flux from the NS surface (Romani 1992), ensure that the BH LMXB systems will be disk instability soft X-ray transients (cf., Whitehurst 1988; Mineshige et al. 1990; see also the contribution of Lasota to these proceedings).

\section{Odd Endpoints}

To summarize the consequences of the evolutionary differences in Section 3, we note that certain unusual classes of $\mathrm{X}$-ray binaries may be found containing BH. First when the primary mass loss is sufficiently severe to allow direct evolution to a WR phase and silent collapse, a distant lower-mass secondary can remain bound without ever having experienced significant mass transfer from the primary. When this star in turn evolves, it can in fact initiate transfer to the primary after it running some distance up the giant branch. A few of these giant-fed, steady bright $\mathrm{BH}$ systems might be found in the inner galaxy (Romani 1992).

The second interesting product is the result of the 'Reverse Mass Transfer' scenario above, leading to a mildly recycled pulsar, with a $\mathrm{BH}$ from the lower-mass secondary star. The BH X-ray binaries should also have a significant number of 'Intermediate-Mass' donors. For NS there should be only a handful of such systems, like Her X-1, in the Galaxy. Presumably, these will be disk transients in many cases. Finally, there should be a surprisingly large number of low-mass X-ray binaries with black holes among the soft $\mathrm{X}$-ray disk transients. The present identifications of A 0620-00, V404 Cyg and Nova Muscae 1991 represent only a small fraction of a large population undergoing steady slow mass transfer and outburst phase accretion.

\section{Population Estimates and Conclusions}

What exactly should we see and how many of the unusual systems above can be hidden in the present XRB and Nova catalogs? The solution is to model the processes above for a full range of progenitor systems. Comparison with observed samples will refine our understanding of the evolutionary processes. Computations of this sort have been made by Tutukov \& Yungel'son (1993), Narayan et al. (1991), Romani (1992, 1994) and Lipunov et al. (1994). Largely these sums are directed at matching the NS X-ray binary and radio pulsar binary populations, but their extrapolation to the $\mathrm{BH}$-producing regime provides some insights. Other population synthesis were presented at a poster by Kalogera \& Tauris at this Symposium, but again the results concentrate on the NS systems. In general, the conclu- 


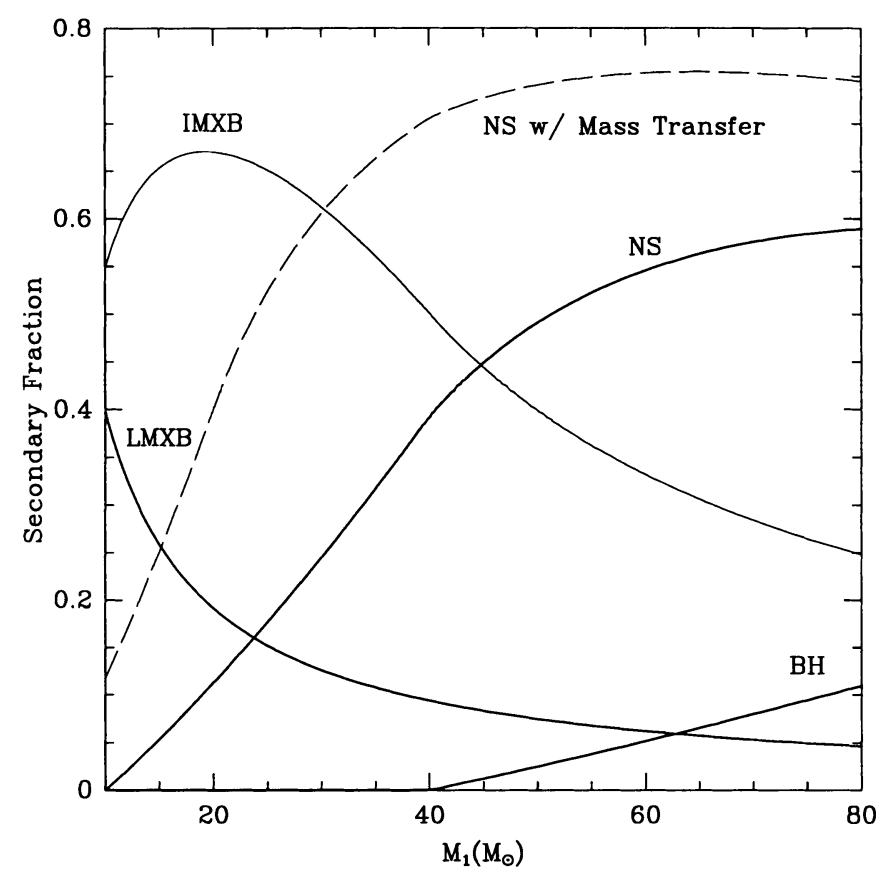

Figure 1. Fraction of secondaries by type, for IMF secondary distribution $q>0.25$, flat in $\log (q)$ below. Primary mass transfer may result in more NS-producing secondaries (dashed line).

sions of these studies are similar, although a more modern treatment of the decimation at supernova events is needed in some cases.

Rough results representative of these calculations can be summarized as follows. The normalization of the star formation rate and the initial IMF produces $\sim 10^{8} \mathrm{NS}$ and $\sim 310^{6} \mathrm{BH}$ during the lifetime of the Galaxy; roughly $310^{5}$ of the former are active radio pulsars, of which about 500 have been detected. The distribution of the mass ratio $q=M_{2} / M_{1}$ in the primordial binary population is presently uncertain: observational evidence for secondaries distributed like the IMF exists for modest $q$ and primaries of moderate mass. For smaller $q$ the number of secondaries probably flattens while for very massive primaries, more equal mass ratios may be preferred. Taking, for example, an IMF secondary distribution to $q=0.25$ and flat in $\log (q)$ thereafter, gives the secondary breakdown shown in Fig. 1; this distribution is particularly uncertain for large $M_{1}$.

After evolving an initial population, typical galactic numbers found for binaries transferring mass in the $\mathrm{X}$-ray producing phase are listed in $\mathrm{Ta}$ ble 1 . Here $\dot{J}$ indicates systems driven by angular-momentum losses, while $\dot{N}$ indicates mass transfer driven by nuclear evolution at longer $P_{\mathrm{b}}$. The 
TABLE 1. Galactic Numbers of NS and Black-Hole Binary Systems

\begin{tabular}{cll}
\hline X-Ray Binary Phase & Active End Phase & $N_{\text {Gal }}$ \\
\hline NS - LMXB & & 100 \\
- IMXB & & $1-3$ \\
- MXRB & NS - NS & 30 \\
& PSR - NS (detected) & $10^{4}$ \\
& & $10^{3}(\sim 500 \dot{J} / 500 \dot{N})$ \\
BH - LMXB & & 10 \\
- LMXB(WR) & & 300 \\
- IMXB & & 3 \\
- HMXB & & $310^{5}$ \\
& BH-NS & $\sim 1 / 2$ \\
\hline
\end{tabular}

low-mass and intermediate-mass systems should in most cases be transients (cf., papers above for details). For the high-mass systems, it is also useful to estimate the number of active pulsar binaries produced. Normalizing to the observed pulsar sample, these computations give roughly $10^{4}$ NS-PSR binaries produced by the HMXB, of which $\sim 5$ should be present in today's pulsar sample. The high-mass BH systems will produce $\sim 310^{5} \mathrm{BH}-\mathrm{NS}$ binaries over the lifetime of the Galaxy, of which $\sim 10^{3}$ are active pulsars. Narayan et al. (1991) and Lipunov et al. (1994) estimate that $\lesssim 1$ of these objects should be present in today's pulsar sample, so future prospects look bright.

The identification of black-hole candidates in the soft X-ray transients has spurred a re-appraisal of the evolutionary channels that can generate black-hole binaries. Interestingly, sums that duplicate the NS XRB population fairly well predict some surprises in the $\mathrm{BH}$ sample. Given the uncertain evolutionary processes and the substantial extrapolation of the presumed binary progenitors from the observed galactic binaries, these results are still quite uncertain. When more $\mathrm{BH}$ candidates are carefully characterized and matched by detailed evolutions, our evolutionary assumptions can merit greater trust. A match to the population statistics and the properties of the candidate systems is also absolutely essential for any satisfactory description. An increase in the present sample by about a factor of 3 , which should occur over the next several years in the case of low-mass black-hole transients, will allow significant progress. If in the process we discover a few of the other unusual end-states predicted by the population synthesis models, then these evolutionary modeling exercises will have been a success. 
Acknowledgements. This work was supported in part by NASA grant NAGW-2963 and the Alfred P. Sloan Foundation.

\section{References}

Brown, G.E., Bruenn, S.W. \& Wheeler, J.C. 1992, Com. Astrophys. 13, 153

Chevalier, R.A. 1989, ApJ 346, 847

Chevalier, R.A. 1993, ApJ 411, L33

Cowley, A.P., Crampton, D. \& Hutchings, J.B. 1983, ApJ 272, 118

De Kool, M., Van den Heuvel, E.P.J. \& Pylyser, E. 1987, A\&A 183, 47

Eggleton, P.P. \& Verbunt, F. 1986, MNRAS 220, 13

Lipunov, V.M. et al. 1994, ApJ 423, L121

Lyne, A.G. \& Lorimer, D.R. 1994, Nat 369, 127

Mineshige, S., Kim, S. \& Wheeler, J.C. 1990, ApJ 358, L5

Nomoto, K. \& Kondo, Y. 1991, ApJ 367, L19.

Narayan, R., Piran, T. \& Shemi, A. 1991, ApJ 379, L17

Podsiadlowski, P., Joss, P.C. \& Hsu, J.J.L. 1992, ApJ 391, 246

Romani, R.W. 1992, ApJ 399, 621

Romani, R.W. 1994, in Interacting Binary Stars, A.E. Shafter (Ed.), ASP Conf. Proc. Vol. 56, p. 196

Savonije, G.J. 1983, in Accretion Driven Stellar X-ray Sources, W.H.G. Lewin \& E.P.J. van den Heuvel (Eds.), Cambridge Univ. Press, p. 434

Tutukov, A.V. \& Yungel'son, L.R. 1993, Ast. Rep. 37, 411.

Van den Heuvel, E.P.J. 1993, in Space Sciences with particular emphasis on High Energy Astrophysics, (ESA: ESTEC Noordwijk)

Van den Heuvel, E.P.J. \& Habets, G.M.H.J. 1984, Nat 309, 689.

Webbink, R.F. 1992, in $X$-ray Binaries and Recycled Pulsars, E.P.J. van den Heuvel \& S.A. Rappaport (Eds.), Kluwer Academic Publishers, p. 269

Wheeler, J.C. \& Shields, G.A. 1976, Nat 259, 642.

Whitehurst, R. 1988, MNRAS 232, 35

Woosley, S.E., Langer, N. \& Weaver, T.A. 1993, ApJ 411, 823

Yamaoka, H., Shigeyama, T. \& Nomoto, K. 1993, A\&A 267, 433. 